\title{
REVIEW
}

\section{Dapagliflozin: a Sodium Glucose Cotransporter 2 Inhibitor in Development for Type 2 Diabetes}

Abd A. Tahrani · Anthony H. Barnett

Received: August 4, 2010 / Published online: January 19, 2011

(c) The Author(s) 2010. This article is published with open access at Springerlink.com

\section{ABSTRACT}

Type 2 diabetes mellitus (T2DM) is a growing worldwide epidemic. Patients face lifelong therapy to control hyperglycemia and prevent the associated complications. There are many medications, with varying mechanisms, available for the treatment of T2DM, but almost all target the declining insulin sensitivity and secretion that are associated with disease progression. Medications with such insulin-dependent mechanisms of action often lose efficacy over time, and there is increasing interest in the development of new antidiabetes medications that are not dependent upon insulin. One such approach is through the inhibition of renal glucose reuptake. Dapagliflozin, the first of a class of selective sodium glucose cotransporter 2 inhibitors, reduces renal glucose reabsorption and is currently under development for the treatment of T2DM. Here, we review the literature relating to the preclinical and clinical development of dapagliflozin.

Abd A. Tahrani $(\bowtie) \cdot$ Anthony H. Barnett Heart of England NHS Foundation Trust, Birmingham Heartlands Hospital, Bordesley Green East, Birmingham B9 5SS, UK and University of Birmingham, Birmingham, UK.

Email: a.a.tahrani@bham.ac.uk
Keywords: dapagliflozin; diabetes; SGLT2 inhibitor

\section{INTRODUCTION}

More than 285 million people worldwide have diabetes and this number is estimated to climb to 438 million by $2030 .{ }^{1}$ Type 2 diabetes mellitus (T2DM) is the most common form, accounting for approximately $85 \%$ of all cases. ${ }^{2}$

$\mathrm{T} 2 \mathrm{DM}$ is a progressive disorder characterized by hyperglycemia, peripheral insulin resistance (in most cases), and a decrease in insulin secretion. ${ }^{3,4}$ Development of T2DM is a lengthy multistage process and changes in insulin resistance, insulin secretion, and plasma glucose can be present many years before a diagnosis of T2DM is made. ${ }^{5}$ Increasing insulin resistance (reduced sensitivity of tissues to insulin) generally occurs as the first in this sequence of events and is followed by a compensatory increase in insulin secretion by the pancreatic beta-cells, thereby maintaining close to normal plasma glucose levels. ${ }^{4,6}$ In those individuals who ultimately develop T2DM a combination of beta-cell dysfunction and a reduction in beta-cell mass culminates in decreasing insulin secretion and associated hyperglycemia. By the time 
T2DM is diagnosed 50\% of the beta-cell function may already be lost. ${ }^{7}$ In addition, excessive and inappropriate glucagon secretion is commonly observed in T2DM, which might exacerbate hyperglycemia and complicate management of the disorder. ${ }^{8}$

As a result of the deleterious effects of chronic hyperglycemia the majority of individuals with T2DM will experience microvascular and/or macrovascular complications. These include diabetic retinopathy, nephropathy, neuropathy, peripheral arterial disease, stroke, and coronary artery disease. ${ }^{9}$ The ultimate goal of any pharmacological intervention in T2DM is to limit microvascular and potentially macrovascular complications by maintaining plasma glucose levels within a relatively normal range. ${ }^{10}$ This link between glycemic control and vascular complications was highlighted in the UK Prospective Diabetes Study (UKPDS). Glycated hemoglobin $\mathrm{A}_{1 \mathrm{c}}\left(\mathrm{HbA}_{1 \mathrm{c}}\right)$ levels were used as a determinant of glycemic control, and for every $1 \%$ reduction in $\mathrm{HbA}_{1 \mathrm{c}}$ it was estimated that there would be an associated $37 \%$ risk reduction for microvascular complications and a $21 \%$ reduction in diabetes-related deaths. ${ }^{11}$

\section{CURRENTLY AVAILABLE TREATMENTS}

There is a well-established association between weight gain and T2DM, and the majority of T2DM patients are overweight or obese. While in some countries treatment of T2DM often begins with the introduction of medications in addition to lifestyle modification, in others lifestyle changes are the first course of treatment implemented and are aimed at restoring glycemic control through diet modification and the introduction of a regular exercise program. However, most patients will be unable to maintain normoglycemia for any length of time without pharmacological intervention. Furthermore, due to the progressive nature of T2DM, an intensification of this initial treatment is often required to maintain glycemic control. This can involve the escalation of medication dosage and/or the inclusion of additional antidiabetes medications into the ongoing treatment plan. A wide choice of antidiabetes medications are available, the majority of which target the increasing insulin resistance or decreasing insulin secretion and are listed below.

- Metformin is generally recommended as the first line of treatment in T2DM. It decreases hepatic glucose production, improves glucose clearance through an improvement of hepatic insulin sensitivity, decreases fatty acid oxidation, and increases glucagon-like peptide 1 (GLP-1; an endogenous peptide secreted from the gastrointestinal tract and a potent stimulator of glucose-dependent insulin release) levels. ${ }^{12-15}$

- Sulfonylureas, such as glimepiride and glipizide, inhibit pancreatic beta-cell $\mathrm{K}_{\mathrm{ATP}}$ channels and enhance insulin secretion. ${ }^{16}$

- Thiazolidinediones (TZDs), such as rosiglitazone and pioglitazone, are peroxisome proliferator-activated receptorgamma agonists. They increase the sensitivity of muscle, fat, and liver to endogenous and exogenous insulin indirectly reducing hepatic glucose production by altering adipose tissue lipid metabolism. ${ }^{13}$

- Meglitinides, such as repaglinide and nateglinide, also bind to the beta-cell $\mathrm{K}_{\text {ATP }}$ channel, albeit at a different site, and stimulate insulin secretion. ${ }^{17}$

- GLP-1 mimetics, including exenatide and liraglutide, bind to GLP-1 receptors at several sites including pancreatic beta-cells. ${ }^{18}$ They potentiate meal-related glucose-dependent insulin secretion and glucagon suppression 
and delay gastric emptying resulting in reduced postprandial hepatic glucose production and enhanced peripheral glucose uptake. ${ }^{19}$

- Dipeptidyl peptidase-4 (DPP-4) inhibitors, such as sitagliptin, vildagliptin, and saxagliptin, prevent the degradation of endogenous GLP-1, thereby prolonging its insulinotropic activity. ${ }^{20,21}$

- Amylin mimetics (eg, pramlintide) are synthetic analogs of the beta-cell hormone amylin. ${ }^{22}$ They act by slowing down the movement of food through the intestine and the absorption of glucose from the intestine, reducing postprandial glucose levels. Amylin mimetics also inhibit postprandial glucagon production. ${ }^{16}$

- The alpha-glucosidase inhibitors, such as acarbose, are one of the few classes of antidiabetes agents that do not have an insulin-dependent mechanism of action. They act by reducing the breakdown of oligosaccharides to monosaccharides in the proximal small intestine, thereby lowering postprandial glucose levels. ${ }^{16}$

- Insulin treatment provides glycemic control through direct stimulation of the insulin receptor. ${ }^{16}$

With the continual decline in insulin secretion and sensitivity that occurs as T2DM progresses, medications that rely upon those mechanisms for their activity frequently lose efficacy and, despite the availability of several different classes of antidiabetic agents, up to $60 \%$ of T2DM patients still do not achieve their target glycemic goals. ${ }^{23}$ There is a need, therefore, for orally active antidiabetes medications that act via insulin-independent mechanisms. One such approach currently under clinical investigation is through inhibition of renal glucose reabsorption and the consequent enhancement of urinary glucose excretion.

\section{RENAL GLUCOSE REABSORPTION AND SODIUM GLUCOSE COTRANSPORTER 2 (SGLT2) INHIBITORS}

The role of the kidneys in maintaining normoglycemia, through the filtration and reabsorption of glucose as well as gluconeogenesis, is well established. Every day $180 \mathrm{~L}$ of plasma are filtered through the kidneys and, in normoglycemic individuals, this translates to approximately $180 \mathrm{~g}$ of glucose. ${ }^{24,25}$ Under normal conditions the ability of the kidneys to reabsorb glucose from the glomerular filtrate is extremely effective, with less than $0.5 \mathrm{~g} /$ day of this filtered glucose ultimately appearing in the urine. Under periods of hyperglycemia the amount of filtered glucose reabsorbed increases in proportion to the plasma glucose concentration until the resorptive capacity of the tubules is exceeded, at which point the excess glucose is excreted in urine (glucosuria). ${ }^{26}$

Glucose reabsorption in the renal tubules is accomplished by way of SGLTs that move glucose into the renal epithelial cells. The majority of the glucose is reabsorbed from the glomerular filtrate by SGLT2. ${ }^{24}$ SGLT2 is a high-capacity, low-affinity transporter predominantly expressed in the kidney where it is exclusively found in the brush border membrane of the S1 segment of the proximal tubule. ${ }^{25,27,28}$ The remainder of the glucose is reabsorbed from the filtrate in the distal S3 segment of the renal proximal tubule by the high-affinity, low-capacity glucose transporter sodium glucose cotransporter 1, SGLT1 (Figure 1A). ${ }^{29,30}$ However, while SGLT2 is predominantly expressed in the kidney, SGLT1 is also highly expressed in the small intestine, where it is involved in the transport of glucose across the brush border membrane. ${ }^{30}$ In the renal tubule an electrochemical gradient generated by the $\mathrm{Na}^{+} / \mathrm{K}^{+}$ATPase located in the 
basolateral membrane drives the movement of sodium ions across the luminal membrane and provides the driving force for glucose cotransport (Figure 1B)..$^{24}$

Increasing urinary glucose excretion through an inhibition of glucose reabsorption represents an attractive method of maintaining blood glucose control without the accompanying risk of hypoglycemia seen with those antidiabetes

Figure 1. (A) Diagram of a renal tubule illustrating the distribution of sodium glucose cotransporter 2 (SGLT2) and SGLT1 and the proportion of glucose reabsorption. The majority of glucose is reabsorbed through SGLT2 versus SGLT1. (B) Schematic representation of the renal reabsorption of glucose by SGLT1/2. SGLT2 transports glucose through the tubular epithelial cells. Glucose concentrated inside the cell is then transported across the basolateral membrane and into the peritubular capillary by glucose transporter 2 (GLUT2). Reproduced with permission.

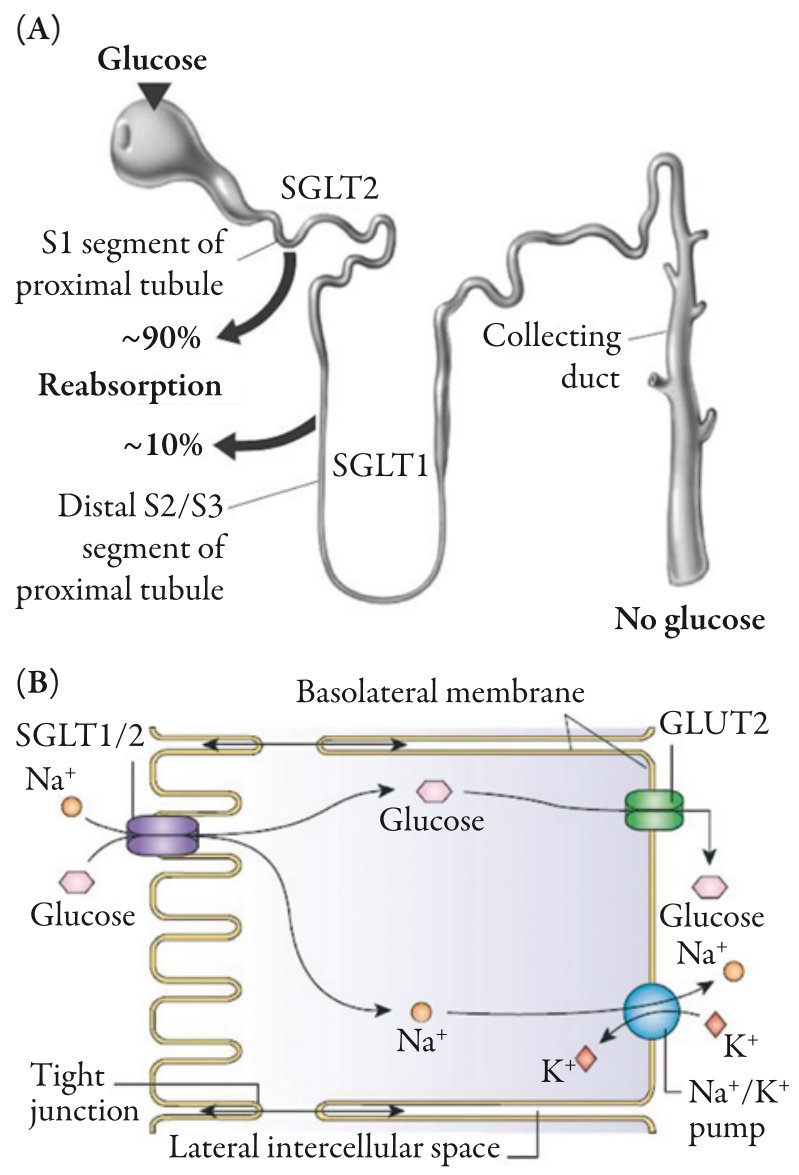

medications that increase insulin secretion. In addition, the caloric loss associated with the excreted glucose may be anticipated to cause weight loss.

The concept of normalizing glucose levels through an increase in urinary glucose excretion is not a new one. The antihyperglycemic properties of the glucosuric agent phlorizin, an SGLT inhibitor derived from apple tree bark, have been known for many years. However, clinical use of phlorizin was not feasible due to nonselectivity. In addition, phlorizin had limited oral bioavailability due to the degradation of an $O$-glucoside linkage by gastrointestinal betaglucosidases. ${ }^{31,32}$

In the quest for a more attractive clinical candidate a number of specific inhibitors of SGLT2 have been developed. Several are undergoing late phase clinical testing for T2DM, eg dapagliflozin, canagliflozin, ASP1941, LX4211, and BI10773. ${ }^{33}$ Two further SGLT2 inhibitors that displayed promising initial results, sergliflozin and remogliflozin etabonate, were discontinued for a variety of reasons, including nonselectivity, unfavorable pharmaceutical properties, or development of replacement SGLT2 compounds. ${ }^{34-36}$

Dapagliflozin is furthest along in development and is currently in phase 3 trials. For the remainder of this article we will review the preclinical and clinical data available for dapagliflozin.

\section{DAPAGLIFLOZIN}

\section{Preclinical Studies}

In preclinical studies dapagliflozin exhibited potent inhibition of human SGLT2 with an $\mathrm{EC}_{50}$ of $1.1 \mathrm{nM}$ and a 1200-fold selectivity for human SGLT2 over human SGLT1, and contained a beta-glucosidase-resistant C-glucoside in place of the $O$-glucoside linkage, permitting 
oral administration. ${ }^{32,37}$ In both normal and experimentally diabetic rats dapagliflozin induced significant renal glucose excretion. ${ }^{37}$ Normal rats exhibited an improved glucose tolerance profile with a single dose of dapagliflozin and this was associated with reductions in glucose excursions following oral glucose tolerance testing (OGTT). ${ }^{37}$ In two different rat models of diabetes (streptozotocin and Zucker diabetic fatty [ZDF] rats) hyperglycemia was reduced after administration of a single oral dose of dapagliflozin and was observed within 6 hours of dosing. ${ }^{32,37}$ Reductions in both fasting and postprandial glucose levels were maintained in ZDF rats over 2 weeks with once-daily dosing with dapagliflozin. ${ }^{37}$ The promising efficacy, tolerability, and overall favorable absorption, distribution, metabolism, and excretion profile of dapagliflozin led to its clinical evaluation in healthy and T2DM subjects. ${ }^{32}$

\section{Clinical Studies}

\section{Pharmacokinetics}

Single-ascending (SAD) and multipleascending (MAD; 2-week duration) dose studies were performed in healthy and T2DM subjects to evaluate the pharmacokinetic and pharmacodynamic profile of dapagliflozin (2.5$500 \mathrm{mg}$ SAD; $2.5-100 \mathrm{mg} /$ day MAD). ${ }^{38,39}$ After oral administration absorption of dapagliflozin was rapid in both healthy and T2DM participants ( $\mathrm{T}_{\max }$ of approximately 1-2 hours). It demonstrated a half-life of approximately 16 to 17 hours in both populations. ${ }^{38,39}$ Dapagliflozin is highly protein bound and renal excretion was minimal throughout the 2-week studies in both populations. Dapagliflozin is primarily metabolized via uridine diphosphate glucuronosyltransferase 1-9 (UGT1A9) to form the inactive glucuronidated metabolite, dapagliflozin 3-O-glucuronide (BMS-801576), which is excreted in the urine. ${ }^{38}$ Total exposure to dapagliflozin was proportional to dose and similar on day 1 and day 14 in both healthy and T2DM subjects. After 14 days dapagliflozin accumulation was minimal and not different between the healthy and T2DM subjects (accumulation index: 1.20-1.30 and 1.23-1.33, respectively). ${ }^{38,39}$ Thus, the pharmacokinetic profile of dapagliflozin was consistent with a once-daily administration protocol.

\section{Pharmacodynamics}

Renal glucose excretion was enhanced by dapagliflozin in healthy and T2DM individuals in a dose-dependent fashion and reached a plateau at the $20 \mathrm{mg} /$ day dose. Following 2 weeks of daily dapagliflozin dosing, cumulative amounts of urinary glucose ranged from 20 to $55 \mathrm{~g} /$ day in healthy subjects and from 37 to $70 \mathrm{~g} /$ day in T2DM patients (Figure 2). ${ }^{38,39}$

In two separate 12 -week trials once-daily dapagliflozin was administered to T2DM participants who were either treatment naïve (dapagliflozin $2.5-50 \mathrm{mg}$ once daily) ${ }^{40}$ or had ongoing insulin therapy with insulin sensitizers (dapagliflozin 10-20 mg once daily). ${ }^{41}$ A similar

Figure 2. Total cumulative amount of glucose excreted in the urine over 24 hours from type 2 diabetes mellitus patients following 14 days of treatment with either dapagliflozin or placebo. A significant increase in urinary glucose excretion was seen at all doses plateauing at the $25 \mathrm{mg}$ once-daily dose. Reproduced with permission.

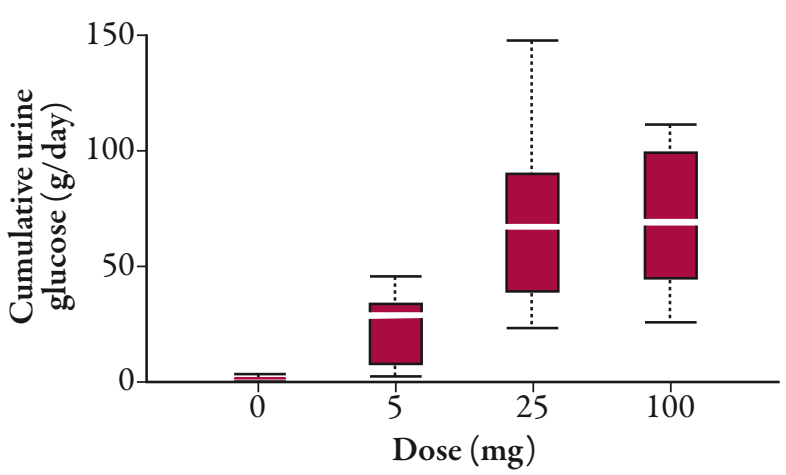


increase in urinary glucose excretion (up to approximately $85 \mathrm{~g} /$ day) was observed at the conclusion of the 12-week treatment period in both populations..$^{40,41}$

\section{Clinical Efficacy in T2DM Patients}

Healthy subjects administered dapagliflozin (2.5-100 mg once daily) for up to 2 weeks exhibited no change in glycemic parameters. ${ }^{38}$ However, T2DM patients administered dapagliflozin (5-100 mg once daily) over the same time period exhibited significant dosedependent reductions in fasting serum glucose at day 13. In addition, improvements in OGTT were also observed on day 13 with all doses of dapagliflozin in the T2DM individuals. ${ }^{39}$

In 12-week studies of once-daily dapagliflozin significant reductions in glucose excursions were seen in T2DM patients following OGTT, as well as dose-proportional reductions in fasting plasma glucose. Significant improvements in $\mathrm{HbA}_{1 \mathrm{c}}$ levels were also seen with dapagliflozin in T2DM subjects over the 12-week dosing period. ${ }^{40,41} \mathrm{~A}-0.55 \%$ to $-0.90 \%$ change in $\mathrm{HbA}_{1 \mathrm{c}}$ was seen following 12 weeks of dapagliflozin treatment (2.5-50 mg once daily) versus $-0.18 \%$ with placebo in treatment-naïve T2DM patients (baseline mean $\mathrm{HbA}_{1 \mathrm{c}}$ 7.6\% to 8\%). Furthermore, significantly more patients achieved their target glycemic goal of $<7 \% \mathrm{HbA}_{1 \mathrm{c}}$ with $50 \mathrm{mg}$ once daily dapagliflozin (59\%) compared with placebo (32\%) following the 12 -week treatment period. ${ }^{40}$ In T2DM patients currently receiving insulin plus an insulin sensitizer, changes of $-0.61 \%$ to $-0.69 \%$ in $\mathrm{HbA}_{1 \mathrm{c}}$ (baseline mean $8.3 \%$ to $8.5 \%$ ) were observed compared with a mean increase of $0.09 \%$ in the placebo group (Figure 3). Around $63 \%$ of those subjects achieved a $\geq 0.5 \%$ reduction at week 12 in $\mathrm{HbA}_{1 \mathrm{c}}$ levels with dapagliflozin (10 and $20 \mathrm{mg}$ once daily) versus $16 \%$ in the placebo group. ${ }^{41}$ This reduction in $\mathrm{HbA}_{1 \mathrm{c}}$ in patients inadequately controlled
Figure 3. Change in mean $\mathrm{HbA}_{1 \mathrm{c}}$ (\%) from baseline over a 12-week treatment period with either dapagliflozin or placebo in type 2 diabetes mellitus subjects with ongoing insulin therapy. A significant reduction in $\mathrm{HbA}_{\mathrm{lc}}$ was seen over 12 weeks with 10 and $20 \mathrm{mg}$ once-daily dapagliflozin. DAPA=dapagliflozin; $\mathrm{HbA}_{1 \mathrm{c}}=$ hemoglobin $\mathrm{A}_{1 \mathrm{c}}$; INS=insulin; LOCF=last observation carried forward; PLA=placebo. Copyright 2009 American Diabetes Association. From Diabetes Care, Vol. 32, 2009; 1656-1662. Reprinted with permission from The American Diabetes Association.

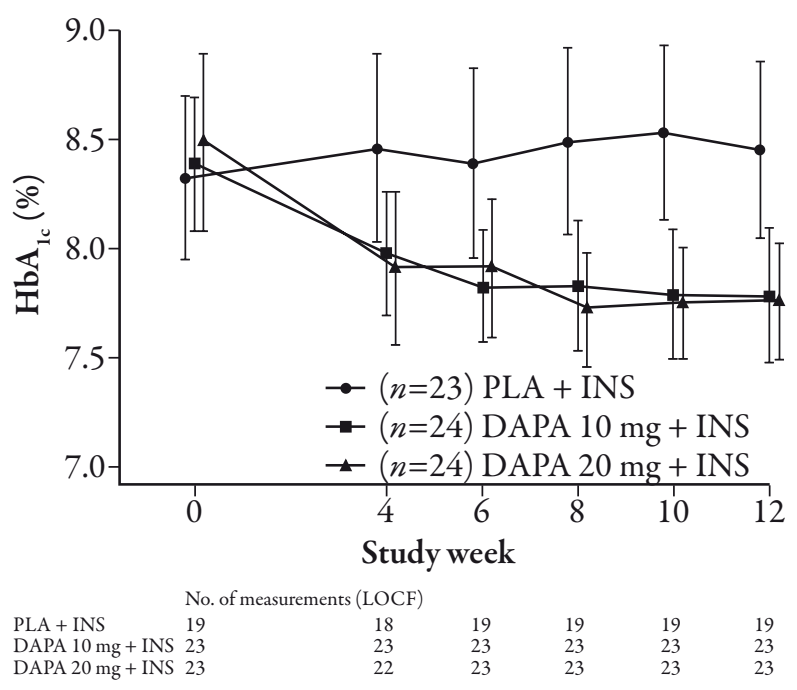

on insulin has been shown to be maintained following 24 and 48 weeks of dapagliflozin treatment. ${ }^{42,43}$ A significant reduction in $\mathrm{HbA}_{1 \mathrm{c}}$ of $-0.75 \%$ to $-0.90 \%$ (mean baseline $\left.\mathrm{HbA}_{1 \mathrm{c}} 8.5 \%\right)$ was observed at 24 weeks with dapagliflozin compared with placebo $(-0.3 \%) .{ }^{42}$ At 48 weeks a change of $-0.74 \%$ to $-0.94 \%$ with $\mathrm{HbA}_{1 \mathrm{c}}$ (baseline mean approximately $8.5 \%$ ) was recorded with dapagliflozin (2.5-10 $\mathrm{mg}$ once daily) compared with $-0.43 \%$ in the placebo group. ${ }^{43}$ Dapagliflozin also reduced the rate of insulin uptitration and study discontinuation as a result of a lack of glycemic control following 48 weeks of treatment in patients with T2DM. ${ }^{44}$ In comparison, the change in mean $\mathrm{HbA}_{1 \mathrm{c}}$ from baseline mean $(8.41 \%)$ seen with the GLP-1 mimetic exenatide in T2DM patients inadequately controlled on insulin was $-1.71 \%$ with exenatide versus $-1.00 \%$ with placebo 
over 30 weeks. ${ }^{45}$ Owing to differences between the trial designs it is not possible to draw direct comparisons between the trials, although it is notable that while the reduction in $\mathrm{HbA}_{1 \mathrm{c}}$ with exenatide was numerically greater than observed with dapagliflozin the reduction in $\mathrm{HbA}_{1 \mathrm{c}}$ in the placebo group was also larger than that seen in the dapagliflozin trial.

When administered for 24 weeks as a monotherapy to treatment-naïve T2DM patients dapagliflozin (2.5-10 mg once daily) produced dose-dependent reductions in $\mathrm{HbA}_{1 \mathrm{c}}$ levels from a baseline mean of $7.8 \%$ to $8.0 \%$ that were statistically significant with the $5 \mathrm{mg}$ and 10 $\mathrm{mg}$ doses $(-0.77 \%$ and $-0.89 \%$, respectively) versus $-0.23 \%$ with placebo. ${ }^{46}$ In patients with ongoing metformin therapy there was a $-0.67 \%$ to $-0.84 \%$ change in $\mathrm{HbA}_{1 \mathrm{c}}$ from a baseline mean of $7.92 \%$ to $8.17 \%$ with dapagliflozin (2.5-10 mg once daily) at 24 weeks as compared with $-0.30 \%$ in the placebo group.$^{47}$ Furthermore, in a 24-week study evaluating the effectiveness of dapagliflozin in patients with T2DM inadequately controlled on the sulfonylurea glimepiride, significant reductions in $\mathrm{HbA}_{1 \mathrm{c}}$ from the baseline mean $(8.07 \%$ to $8.15 \%)$ of $-0.58 \%$ to $-0.82 \%$ with dapagliflozin $(2.5-10 \mathrm{mg}$ once daily) were observed compared with $-0.13 \%$ with placebo. ${ }^{48}$

In the longest reported trial with dapagliflozin so far (52 weeks) reductions in $\mathrm{HbA}_{1 \mathrm{c}}$ with dapagliflozin from baseline mean (approximately 7.72\%) were reported in patients poorly controlled with metformin. The adjusted mean change from baseline was $-0.52 \%$ with dapagliflozin ( $\leq 10 \mathrm{mg}$ once daily) compared with a similar reduction of $-0.52 \%$ with the sulfonylurea glipizide after 52 weeks of treatment. ${ }^{49}$

Total body weight loss occurred in all groups in the 12-week study in treatment-naïve T2DM patients. The reduction in body weight was continuous across the dosing period and ranged from $-2.5 \%$ to $-3.4 \%$ at 12 weeks and $-1.2 \%$ in placebo although this did not reach statistical significance. ${ }^{40}$ After 12 weeks the change in body weight with dapagliflozin in T2DM patients taking insulin and insulin sensitizers was -4.3 to $-4.5 \mathrm{~kg}$ in the dapagliflozin treatment groups versus $-1.9 \mathrm{~kg}$ with placebo (Figure 4$).{ }^{41}$ Both 24-week and 48-week studies were consistent with this effect of dapagliflozin on weight loss (24 weeks: $-1.67 \mathrm{~kg}$ with $10 \mathrm{mg}$ vs. $0.02 \mathrm{~kg}$ placebo, $\mathrm{P} \leq 0.0001 ; 48$ weeks: $-1.79 \mathrm{~kg}$ with $10 \mathrm{mg}$ vs. $-0.18 \mathrm{~kg}) .{ }^{42,43}$ In treatment-naïve T2DM patients, decreases of up to $3.3 \mathrm{~kg}$ were observed after 24 weeks of treatment with dapagliflozin and decreases of $2.2 \mathrm{~kg}$ were evident in the placebo group, although this was not reported as being significant. In metformin-treated T2DM patients, reductions of up to $3.0 \mathrm{~kg}$ in body

Figure 4. Changes from baseline in mean body weight over 12 weeks of treatment with dapagliflozin (DAPA) or placebo (PLA) in subjects with ongoing insulin (INS) therapy. A trend towards continuous and greater weight loss was seen over 12 weeks with 10 and $20 \mathrm{mg}$ once-daily dapagliflozin versus placebo. LOCF=last observation carried forward. Copyright 2009 American Diabetes Association. From Diabetes Care, Vol. 32, 2009; 1656-1662. Reprinted with permission from The American Diabetes Association.

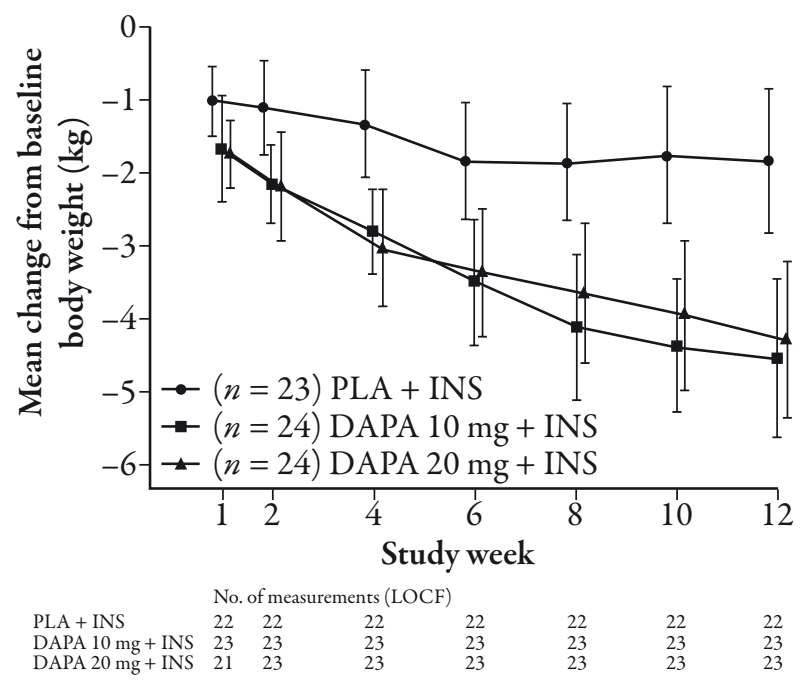


weight were seen with dapagliflozin (2.5-10 mg once daily) as compared with $0.9 \mathrm{~kg}$ in the placebo group following 24 weeks of treatment, with similar reductions following 52 weeks of treatment. ${ }^{46,47,49}$

\section{Tolerability and Side Effects}

In T2DM patients adverse events observed in the dapagliflozin treatment and placebo groups were similar in frequency and were generally mild in nature whether for patients who were treatment naïve or for those receiving ongoing metformin or insulin plus insulin sensitizer therapy. ${ }^{40-47} \mathrm{Few}$ cases of hypoglycemia were observed and these were generally mild, self-limiting, and occurred with a similar frequency in the placebo group; none were severe. ${ }^{40-47}$

A mild diuretic effect of dapagliflozin was observed at week 12 in the treatment-naïve patients (an increase in urinary output of up to $470 \mathrm{~mL} /$ day above baseline compared with a $112 \mathrm{~mL} /$ day decrease in placebo). In those T2DM patients taking insulin plus oral insulin sensitizers there was an increase in urine output of up to $444 \mathrm{~mL} /$ day above baseline compared with $255 \mathrm{~mL} /$ day with placebo (although the difference was not reported as being significant). ${ }^{40,41}$ Consistent with this observation, dapagliflozin appears to be associated with a mild reduction in mean blood pressure with no evidence of orthostatic hypotension. ${ }^{40,41}$

The long-term effects of dapagliflozin on renal function are as yet unknown, as are the effects of dapagliflozin when used in cases of compromised renal function or when coadministered with certain antihypertensive drug classes that may affect renal physiology. However, no clinically relevant changes in glomerular filtration rate have been reported with dapagliflozin treatment. ${ }^{39-41}$ Owing to the inhibition of the cotransportation of sodium and glucose, dapagliflozin administration may result in an increase in urinary sodium.
Acute transient increases in urine sodium have been observed with dapagliflozin treatment. ${ }^{39}$ Median changes from baseline in urinary sodium excretion were $+34.7,+40.2$, and $+48.0 \mathrm{mEq}$ for the 5,25 , and $100 \mathrm{mg}$ dapagliflozin treatment groups, and $-15.1 \mathrm{mEq}$ for the placebo group over the initial 24 hours. With continuing daily administration this increase in sodium excretion appeared to normalize, with median changes from baseline at day 13 of $+1.8,+8.9$, and $-5.7 \mathrm{mEq}$ for the 5,25 , and $100 \mathrm{mg}$ dapagliflozin dose groups and $+16.4 \mathrm{mEq}$ for placebo. Despite the reported transient increase in urinary sodium excretion there is no evidence to suggest that this is reflected in any changes in serum sodium levels. ${ }^{39}$

Although dapagliflozin has been associated with increases in urine volume, serum magnesium, serum phosphate, and serum uric acid these changes were still within normal physiological ranges and there was little evidence to suggest that dapagliflozin would produce clinically significant changes in electrolyte balance.

Because it was hypothesized that increasing urinary glucose may promote microbial growth, potential urinary tract infections (UTIs) and genital infections were carefully monitored. While the frequency of reported UTIs was similar between the dapagliflozin and placebo groups over the 12-week time period, symptoms suggestive of genital infections (in particular vulvocandidiasis) were reported more frequently in the groups administered dapagliflozin..$^{40,41}$

In the 24-week studies, signs and symptoms suggestive of genital infection were reported in up to $13 \%$ of T2DM patients who were either treatment naïve or who were inadequately controlled with metformin as compared with $1.3 \%$ and $5 \%$ in the respective placebo groups. ${ }^{46,47}$ No increased rate of discontinuation occurred as a result of these signs and symptoms suggestive of UTIs or genital infections. ${ }^{39,41-43,46-48}$ It is not 
known at this time how these observations in controlled trials will translate into infection rates in everyday clinical practice until more widespread experience is gained over a longer time frame.

Additional data are necessary to fully elucidate the long-term effects of glucose excretion induced by dapagliflozin. It is noteworthy, however, that people with a condition known as familial renal glucosuria (FRG), arising primarily from a mutation of the gene encoding for the SGLT2 protein (SLC5A2), live normal healthy lives, with no adverse events, despite the consistently high levels of urinary glucose.$^{50}$ Experience with FRG is based on a very limited number of cases, and caution should be exercised when making comparisons with the general diabetic population. In these patients glucosuria can range from $<1$ to $>150$ $\mathrm{g} / 1.73 \mathrm{~m}^{2}$ per day. While FRG patients generally have few clinical complications polyuria and enuresis were observed in a single case study and were followed later by a slight delay in growth and pubertal maturation. ${ }^{51}$ Other reported complications with FRG include episodes of dehydration and ketosis as well as urinary tract infections and natriuresis. ${ }^{50}$

However, unlike FRG, T2DM is associated with impaired immune responses, and patients with T2DM may have side effects relating to elevated urinary glucose levels. Furthermore, while glucose-induced diuresis does not appear to lead to dehydration in the majority of cases of FRG, the mild diuretic properties of dapagliflozin may require careful monitoring to ensure adequate fluid intake and warrants further investigation. One episode of renal failure did occur during treatment with 10 mg dapagliflozin in a patient concomitantly being treated with insulin and several antihypertensive medications (enalapril, carvedilol, and furosemide). The patient discontinued dapagliflozin as a result of dehydration and prerenal azotemia. The condition was resolved with oral rehydration and the withholding of enalapril and furosemide. ${ }^{41}$

\section{SUMMARY AND THERAPEUTIC APPLICATIONS}

$\mathrm{T} 2 \mathrm{DM}$ is a progressive disease with a significant impact on patients, society, and healthcare systems. The aim of treatment is to achieve near normal glycemia in order to prevent the development or slow the progression of microvascular and possibly macrovascular complications. Currently available antidiabetes treatments have many limitations, including hypoglycemia (sulfonylureas, meglitinides, and insulin), weight gain (sulfonylureas, meglitinides, TZDs, and insulin), heart failure (TZDs), gastrointestinal side effects (alphaglucosidase inhibitors, metformin), and the need for multiple injections and/or self-monitoring (insulin, amylin mimetics, and GLP-1 analogs). Furthermore, due to the progressive nature of the disease, most patients will ultimately require multiple antidiabetes treatments to achieve glycemic targets. Hence, there is a need for new antidiabetes treatments that produce a sustainable impact on glycemic control with low risk for hypoglycemia and weight loss, and minimal need for self-monitoring. SGLT2 inhibitors are a novel class of antidiabetes therapy that are taken orally, result in improvements in glycemic parameters with a low risk for hypoglycemia, and are associated with weight loss. They have the potential to harness what has long been considered a manifestation of diabetes, glucosuria, and turn it into a therapeutic strategy. However, like other newer antidiabetes treatments, this class lacks long-term (including cardiovascular) safety data. 
With such a plethora of options to treat patients with T2DM, the decision to use a particular drug, or combination of drugs, in a particular patient should be individualized based on the patient's specific risk-benefit balance and not solely upon a drug's perceived ability to lower $\mathrm{HbA}_{1 \mathrm{c}}$. Metformin (in addition to lifestyle changes) remains a well-established first-line treatment for patients with T2DM, due to good long-term safety data, lack of hypoglycemia or weight gain, and evidence for cardiovascular protection. However, as metformin does not halt the progression of the disease, patients with T2DM are likely to need additional antidiabetic medications administered alone or in combination with metformin. Should SGLT2 inhibitors, such as dapagliflozin, prove to have an acceptable safety profile they may have the potential to be administered alone or in combination with metformin or insulin. The lack of long-term (including cardiovascular) safety data and other outcome data may limit their use initially to specific/defined low-risk patient groups.

\section{CONCLUSION}

In conclusion, dapagliflozin has the potential to be a useful addition to currently available antidiabetes treatments as it lowers fasting and postprandial glucose levels, improves glycemic control, and causes weight loss with a low risk of hypoglycemia. However, data regarding longterm safety including urinary tract infection, genital infection, and cardiovascular safety are needed, and its place in the algorithm of T2DM management is still to be determined.

\section{ACKNOWLEDGMENTS}

Dr. Karen Pemberton of PPSI (a PAREXEL company) provided writing assistance, which was funded by AstraZeneca and Bristol-Myers Squibb.

AAT is a research training fellow supported by the National Institute for Health Research. The views expressed in this publication are those of the author(s) and not necessarily those of the NHS, the National Institute for Health Research, or the Department of Health.

AHB has received honoraria for lectures and advisory work and research funding from Servier, MSD, Novartis, Takeda, Glaxo Smith Kline, BMS/ AstraZeneca, Eli Lilly, Novo Nordisk, Roche, and Sanofi-Aventis.

Open Access. This article is distributed under the terms of the Creative Commons Attribution Noncommercial License which permits any noncommercial use, distribution, and reproduction in any medium, provided the original author(s) and source are credited.

\section{REFERENCES}

1. International Diabetes Federation. IDF Diabetes Atlas. Available at: www.diabetesatlas.org/book/ export/html/36. Accessed April 21, 2010.

2. World Health Organization. Global Strategy on Diet, Physical Activity and Health. Available at: www.who.int/dietphysicalactivity/publications/ facts/obesity/en/. Accessed April 21, 2010.

3. Weyer C, Tataranni PA, Bogardus C, Pratley RE. Insulin resistance and insulin secretory dysfunction are independent predictors of worsening of glucose tolerance during each stage of type 2 diabetes development. Diabetes Care. 2001;24:89-94.

4. DeFronzo RA. Pathogenesis of type 2 diabetes mellitus. Med Clin North Am. 2004;88:787-835.

5. Tabak AG, Jokela M, Akbaraly TN, Brunner EJ, Kivimaki M, Witte DR. Trajectories of glycaemia, insulin sensitivity, and insulin secretion before diagnosis of type 2 diabetes: an analysis from the Whitehall II study. Lancet. 2009;373:2215-2221.

6. Bonner-Weir S. Islet growth and development in the adult. J Mol Endocrinol. 2000;24:297-302. 
7. Goldstein BJ. Insulin resistance as the core defect in type 2 diabetes mellitus. Am J Cardiol. 2002;90:3G-10G.

8. Jiang G, Zhang BB. Glucagon and regulation of glucose metabolism. Am J Physiol Endocrinol Metab. 2003;284:E671-E678.

9. Nichols GA, Arondekar B, Herman WH. Complications of dysglycemia and medical costs associated with nondiabetic hyperglycemia. Am J Manag Care. 2008;14:791-798.

10. Fowler MJ. Microvascular and macrovascular complications of diabetes. Clin Diabetes. 2008;26:77-82.

11. Stratton IM, Adler AI, Neil HA, et al. Association of glycaemia with macrovascular and microvascular complications of type 2 diabetes (UKPDS 35): prospective observational study. BMJ. 2000;321:405-412.

12. Effect of intensive blood-glucose control with metformin on complications in overweight patients with type 2 diabetes (UKPDS 34). UK Prospective Diabetes Study (UKPDS) Group. Lancet. 1998;352:854-865.

13. Natali A, Ferrannini E. Effects of metformin and thiazolidinediones on suppression of hepatic glucose production and stimulation of glucose uptake in type 2 diabetes: a systematic review. Diabetologia. 2006;49:434-441.

14. Bailey CJ, Turner RC. Metformin. N Engl J Med. 1996;334:574-579.

15. Mannucci E, Tesi F, Bardini G, et al. Effects of metformin on glucagon-like peptide-1 levels in obese patients with and without type 2 diabetes. Diabetes Nutr Metab. 2004;17:336-342.

16. Nathan DM, Buse JB, Davidson MB, et al. Medical management of hyperglycemia in type 2 diabetes: a consensus algorithm for the initiation and adjustment of therapy: a consensus statement of the American Diabetes Association and the European Association for the Study of Diabetes. Diabetes Care. 2009;32:193-203.

17. Hu S. Interaction of nateglinide with K(ATP) channel in beta-cells underlies its unique insulinotropic action. Eur J Pharmacol. 2002;442:163-171.

18. Drucker DJ. Biologic actions and therapeutic potential of the proglucagon-derived peptides. Nat Clin Pract Endocrinol Metab. 2005;1:22-31.
19. Tahrani AA, Piya MK, Kennedy A, Barnett AH. Glycaemic control in type 2 diabetes: targets and new therapies. Pharmacol Ther. 2010;125:328-361.

20. Palalau AI, Tahrani AA, Piya MK, Barnett AH. DPP4 inhibitors in clinical practice. Postgrad Med. 2009;121:70-100.

21. Tahrani AA, Piya MK, Barnett AH. Saxagliptin: a new DPP-4 inhibitor for the treatment of type 2 diabetes mellitus. Adv Ther. 2009;26:249-262.

22. Schmitz O, Brock B, Rungby J. Amylin agonists: a novel approach in the treatment of diabetes. Diabetes. 2004;53(suppl. 3):S233-S238.

23. Del Prato S, Felton AM, Munro N, Nesto R, Zimmet $\mathrm{P}$, Zinman B. Improving glucose management: ten steps to get more patients with type 2 diabetes to glycaemic goal. Int J Clin Pract. 2005;59:1345-1355.

24. Wright EM, Hirayama BA, Loo DF. Active sugar transport in health and disease. J Intern Med. 2007;261:32-43.

25. Wright EM. Renal $\mathrm{Na}(+)$-glucose cotransporters. Am J Physiol Renal Physiol. 2001;280:F10-F18.

26. Marsenic O. Glucose control by the kidney: an emerging target in diabetes. Am J Kidney Dis. 2009;53:875-883.

27. Chen J, Feder J, Neuhaus I, Whaley JM. Tissue expression profiling of the sodium-glucose cotransporter (SGLT) family: implication for targeting SGLT2 in type 2 diabetes patients. Diabetes. 2008;57(suppl. 1):A682. Abstract 2493.

28. Kanai Y, Lee WS, You G, Brown D, Hediger MA. The human kidney low affinity $\mathrm{Na}$ /glucose cotransporter SGLT2. Delineation of the major renal reabsorptive mechanism for D-glucose. J Clin Invest. 1994;93:397-404.

29. Wood IS, Trayhurn P. Glucose transporters (GLUT and SGLT): expanded families of sugar transport proteins. Br J Nutr. 2003;89:3-9.

30. Lee YJ, Lee YJ, Han HJ. Regulatory mechanisms of $\mathrm{Na}+$ /glucose cotransporters in renal proximal tubule cells. Kidney Int. 2007;72:S27-S35.

31. Brooks AM, Thacker SM. Dapagliflozin for the treatment of type 2 diabetes. Ann Pharmacother. 2009;43:1286-1293.

32. Meng W, Ellsworth BA, Nirschl AA, et al. Discovery of dapagliflozin: a potent, selective renal sodiumdependent glucose cotransporter 2 (SGLT2) inhibitor for the treatment of type 2 diabetes. J Med Chem. 2008;51:1145-1149. 
33. Chao EC, Henry RR. SGLT2 inhibition-a novel strategy for diabetes treatment. Nat Rev Drug Discov. 2010;9:551-559.

34. Dobbins R, Kapur A, Kapitza C, O'Connor-Semmes R, Tao W, Hussey EK. Remogliflozin etabonate, a selective inhibitor of the sodium-glucose transporter 2 (SGLT2) reduces serum glucose in type 2 diabetes mellitus (T2DM) patients. Diabetes. 2009;58(suppl. 1). Abstract.

35. Hussey EK, Kapur A, O'Connor-Semmes R, Tao W, Poo J, Dobbins R. Safety, pharmacokinetics and pharmacodynamics of remogliflozin etabonate (SGLT2 inhibitor) and metformin when coadministered in type 2 diabetes mellitus (T2DM) patients. Diabetes. 2009;58(suppl. 1). Abstract.

36. Hussey E, Clark RV, Amin DM, et al. Early clinical studies to assess the safety, tolerability, pharmacokinetics and pharmacodynamics of single doses of sergliflozin, a novel inhibitor or renal glucose reabsorption, in healthy volunteers and subjects with type 2 diabetes mellitus. Diabetes. 2007;56:A49. Abstract.

37. Han S, Hagan DL, Taylor JR, et al. Dapagliflozin, a selective SGLT2 inhibitor, improves glucose homeostasis in normal and diabetic rats. Diabetes. 2008;57:1723-1729.

38. Komoroski B, Vachharajani N, Boulton D, et al. Dapagliflozin, a novel SGLT2 inhibitor, induces dose-dependent glucosuria in healthy subjects. Clin Pharmacol Ther. 2009;85:520-526.

39. Komoroski B, Vachharajani N, Feng Y, Li L, Kornhauser D, Pfister M. Dapagliflozin, a novel, selective SGLT2 inhibitor, improved glycemic control over 2 weeks in patients with type 2 diabetes mellitus. Clin Pharmacol Ther. 2009;85:513-519.

40. List JF, Woo V, Morales E, Tang W, Fiedorek FT. Sodium-glucose cotransport inhibition with dapagliflozin in type 2 diabetes. Diabetes Care. 2009;32:650-657.

41. Wilding JPH, Norwood P, T'joen C, Bastien A, List JF, Fiedorek FT. A study of dapagliflozin in patients with type 2 diabetes receiving high doses of insulin plus insulin sensitizers. Applicability of a novel insulin-independent treatment. Diabetes Care. 2009;32:1656-1662.

42. Wilding JPH, Woo V, Soler NG, Pahor A, Sugg J, Parikh S. Dapagliflozin in patients with type 2 diabetes poorly controlled on insulin therapy-efficacy of a novel insulin-independent treatment. Diabetes. 2010;59(suppl. 1):A21-A22. Abstract 78-OR.

43. Wilding JPH, Woo V, Soler NG, Pahor A, Sugg J, Parikh S. Sustained effectiveness of dapagliflozin over 48 weeks in patients with type 2 diabetes poorly controlled with insulin. Diabetes. 2010;59(suppl. 1). Abstract 0021-LB.

44. Soler NG, Wilding JPH, Woo V, et al. Dapagliflozin lowered rate of insulin uptitration/study discontinuation from lack of glycaemic control in 48-week study of type 2 diabetes patients poorly controlled on insulin therapy. Diabetologia. 2010;53(suppl. 1):S348-S349.

45. Bergenstal RM, Buse J, Glass L, et al. Exenatide added to insulin glargine-treated patients with type 2 diabetes provided excellent fasting and postprandial control with weight loss and no increased risk of hypoglycaemia. Diabetologia. 2010;53(suppl. 1):S37. Abstract.

46. Ferrannini E, Ramos SJ, Salsali A, Tang W, List JF. Dapagliflozin monotherapy in type 2 diabetic patients with inadequate glycemic control by diet and exercise: a randomized, double-blind, placebo-controlled, phase III trial. Diabetes Care. 2010;33:2217-2224.

47. Bailey CJ, Gross JL, Pieters A, Bastien A, List JF. Effect of dapagliflozin in patients with type 2 diabetes who have inadequate glycaemic control with metformin: a randomised, double-blind, placebo-controlled trial. Lancet. 2010;375:22232233.

48. Strojek K, Hruba V, Elze M, Langkilde A, Parikh S. Efficacy and safety of dapagliflozin in patients with type 2 diabetes mellitus and inadequate glycaemic control on glimepiride monotherapy. Diabetologia. 2010;53(suppl. 1):S347. Abstract.

49. Nauck M, Del Prato S, Rohwedder K, Elze M, Parikh S. Dapagliflozin vs glipizide in patients with type 2 diabetes mellitus inadequately controlled on metformin: 52-week results of a double-blind, randomized, controlled trial. Diabetologia. 2010;53(suppl 1). Abstract.

50. Santer R, Calado J. Familial renal glucosuria and SGLT2: from a mendelian trait to a therapeutic target. Clin J Am Soc Nephrol. 2010;5:133-141.

51. Scholl-Burgi S, Santer R, Ehrich JH. Long-term outcome of renal glucosuria type 0 : the original patient and his natural history. Nephrol Dial Transplant. 2004;19:2394-2396. 